UDK 528.711.11

\title{
PERFORMANCE EVALUATION OF NON-METRIC DIGITAL CAMERA FOR PHOTOGRAMMETRIC APPLICATION
}

\author{
Birutė Ruzgienė \\ Dept of Geodesy and Cadastre, Vilnius Gediminas Technical University, \\ e-mail: Birute.Ruzgiene@ap.vtu.lt
}

Received 1012 2004, accepted 03012005

\begin{abstract}
During the last year amateur low-cost digital cameras are increasingly expected to contribute to the digital photogrammetry. An important aspect of the suitability of these cameras is determination of their geometrical instability. In order to evaluate amateur digital camera performance, small format, low resolution and low-cost CCD camera have been investigated in two considerations: determining inner orientation parameters at different time and estimating accuracy in test field experiments. The calibration results demonstrate the poor stability of such a digital camera. As a result of the instability, amateur cameras have limited possibilities in close-range photogrammetry. However, the investigated camera under certain limited accuracy requirements can be used for low-accuracy photogrammetric application.
\end{abstract}

Keywords: close-range photogrammetry, inner orientation, non-metric camera, calibration, CCD, camera parameter.

\section{Introduction}

For the last three decades the development of analytical photogrammetry has allowed the extensive use of non-metric (amateur) cameras for image acquisition. Cameras, which have no fiducial marks, can be used for photogrammetric application with a considerable difficulty. It needs special computations that permit transformation of amateur imagery.

The digital cameras based on CCD sensors (Charged Coupled Devices) are fast replacing classical film-based cameras. CCD metric cameras such as Kodak Professional DCS460, DC370, DCS210; Rollei Metric d7; Sony Cybershot, etc are used for image acquisition. The major imperfection of CCD cameras is the limited resolution or limited field of view. However, cameras whose resolution is about 15 mega pixels have already been announced. Up-to-date technology is employed for digital image processing. In close-range (terrestrial) photogrammetry CCD camera are mainly applied for surveys of buildings, restoration of architectural monuments, documentation of buildings, reconstruction of the details of traffic accidents, mobile mapping as well as fast and low cost mapping applications from light aircraft or helicopter.

Present-day digital technology can provide nonmetric images by using digital amateur cameras that are relatively widely employed in close-range photogrammetry. The development of a low-cost photogrammetric system using amateur digital camera is one of the important tasks for the non-topographic photogrammetry.

The amateur digital cameras, which have highresolution sensors, are increasingly expected to contribute to the digital photogrammetry. Since 2000 the resolution (number of pixels) of amateur camera has been rapidly increasing. At present, various kinds of amateur digital cameras are on the market. Table shows the number of pixels in some types of amateur cameras.

Number of pixels in amateur digital camera

\begin{tabular}{|l|l|c|}
\hline Manufacturer & Amateur camera type & $\begin{array}{c}\text { Resolution } \\
\text { (Mega pixels) }\end{array}$ \\
\hline \hline FUJI FILM & FinePix2700 & 2,5 \\
\hline CASIO & EX-Z3 & 3,2 \\
\hline CANON & IXY DIGITAL 30 & 3,2 \\
\hline PENTAX & OptioS & 3,2 \\
\hline MINOLTA & DiMAGE Xt & 3,2 \\
\hline OLYMPUS & E-10 & 4,0 \\
\hline SONY & Cyber-shot DSC-F77 & 4,0 \\
\hline SONY & Cyber-shot DSC-T1 & 5,1 \\
\hline MINOLTA & DiMAGE 7 & 5,2 \\
\hline
\end{tabular}

A current trend of amateur digital cameras development is miniaturisation and lightening. Also, mobile phones with high resolution CCD sensor are appearing at present. In these circumstances, various applications of amateur digital cameras (with more than 3 mega pixels) for digital photogrammetric field and incorporating them with telecommunications equipment can be expected.

The drawbacks in the use of small format amateur cameras (non-metric imagery) are the lack of fiducial marks, unknown and instable inner orientation parameters. In order to evaluate an amateur digital camera from the point of view of digital photogrammetic application the instability of inner orientation parameters 
as well as the performance evaluation of amateur, small format and low-cost CCD camera have been investigated.

\section{Some aspects on images orientation}

The reconstruction and transformation of spatial object from photographs needs determination of inner and outer orientation elements. Three independent parameters of inner orientation (image coordinates of the principal point $x_{o}, y_{o}$ and focal distance $c$ define the position of the projection centre relative to the image plane.

Relation between image $(x, y)$ and ground coordinates $(X, Y, Z)$ is expressed by formulae [1]:

$$
\begin{aligned}
& x-x_{0}=-c \frac{r_{11}\left(X-X_{0}\right)+r_{21}\left(Y-Y_{0}\right)+r_{31}\left(Z-Z_{0}\right)}{r_{13}\left(X-X_{0}\right)+r_{23}\left(Y-Y_{0}\right)+r_{33}\left(Z-Z_{0}\right)}, \\
& y-y_{0}=-c \frac{r_{12}\left(X-X_{0}\right)+r_{22}\left(Y-Y_{0}\right)+r_{32}\left(Z-Z_{0}\right)}{r_{13}\left(X-X_{0}\right)+r_{23}\left(Y-Y_{0}\right)+r_{33}\left(Z-Z_{0}\right)},
\end{aligned}
$$

where $X_{0}, Y_{0}, Z_{0}$ are object coordinates of camera position, $r_{i j}$ - elements of the rotation matrix $\boldsymbol{R}$ derived from the elements of relative orientation by rotation.

In relative orientation relationship between the image coordinates $x$ and $y$ and the image coordinates $x^{\prime}$ and $y^{\prime}$ of the normalised image are derived from the collinearity condition [2]:

$$
\begin{aligned}
& x=-c \frac{r_{11} x^{\prime}+r_{21} y^{\prime}-r_{31} c^{\prime}}{r_{13} x^{\prime}+r_{23} y^{\prime}-r_{33} c^{\prime}}, \\
& y=-c \frac{r_{12} x^{\prime}+r_{22} y^{\prime}-r_{32} c^{\prime}}{r_{13} x^{\prime}+r_{23} y^{\prime}-r_{33} c^{\prime}},
\end{aligned}
$$

where $c^{\prime}-$ normalised principal distance.

The inner orientation parameters (image coordinates of the principal point, principal distance, radial and tangent distortions) can be determined by means of calibration: 1) the use of a dense network of measured spatial control points; 2) in camera self-calibration.

The angular relationship between the camera and object space resection can be utilised in camera calibration where coordinates in the object space may be known, but the camera focal length and the lens distortion affecting observed photo coordinates are to be determined.

In the photographs especially from amateur cameras the central projection is deformed by lens distortion. Such influence can be eliminated in a bundle block adjustment with additional parameters (in self-calibration). Lens distortion causes a point's image on imaging surface to be shifted from its true position. The compensation for any point coordinates (if the principal point is 0,0 ) is given by:

$$
x_{c}=x+\Delta r_{x}+\Delta p_{x}, y_{c}=y+\Delta r_{y}+\Delta p_{y},
$$

where $x_{c}, y_{c}$ - corrected image point; $\Delta r_{x}, \Delta r_{y}-x$ and $y$ components of the lens radial corrections; $\Delta p_{x}, \Delta p_{y}-$ decentring lens distortion corrections.

The radial lens distortion is radially symmetric about principal point. A marked point at a radial distance $r$ from the principal point has to be compensating by factor $\Delta r$. The radial lens distortion $\Delta r$ is modelled using odd power polynomial, based on lens design theory:

$$
\Delta r=k_{0} r+k_{1} r^{3}+k_{2} r^{5}+k_{3} r^{7}
$$

where $r=\sqrt{\left(x-x_{P}\right)^{2}+\left(y-y_{P}\right)^{2}}-$ radial distance from the principal point; $k_{0}, k_{1}, k_{2}, k_{3}$-coefficients of the polynomial, solved by least squares adjustment.

Correction for lens distortion is calculated:

$$
x_{c}^{\prime}=x\left(1-\frac{\Delta r}{r}\right), \quad y_{c}^{\prime}=y\left(1-\frac{\Delta r}{r}\right) .
$$

Decentring distortion has tangential and radial asymmetric components. Tangential distortion occurs in a parallel orientation at points across the image and varies as a function of the direction to the point from the principal point. Decentring distortion is due to imperfect centring of the lens components and is much smaller than radial lens distortion, therefore is used for the highest accuracy measurements.

If digital image is to be used, it requires knowledge of the inner orientation parameters as well as a relation between the pixel position and $\eta \xi$-coordinate system. If the pixels are very small, it requires knowing which pixel contains the principal point.

Different methods can overcome the lack of fiducial marks. One of solution for inner orientation of images without fiducials is the measurement of the frame corners $[3,4]$. These corners can define the coordinate system. But when digital image is zooms in, it is difficult to determine the exact position of the corner, so it is need to make redundant measurements at the all format edges. After edge lines have been defined, corners are computed by intersection of lines. Usually $x$-axis has to be parallel to the lower frame edge.

Digital amateur cameras are mechanically unstable and must be calibrated at short intervals on the test field. Self-calibration approach can be applied for taking photography with these cameras as well [5]. The parameters of camera inner orientation are determined in laboratory measurements taking photography of a particular test field.

\section{Evaluation of amateur digital camera performance}

The non-metric, unstable, not professional and lowcost digital camera JENOPTIK JD11 has been selected for investigation. Main specifications of digital amateur camera: resolution (image size) $-640 \times 480$ pixels (VGA); film speed index - ISO 40; colour - 24 bits; memory card - store up to 18 images; flash - 2,5 m at maximum; 
self timer - $10 \mathrm{sec}$; focus range $-2,5 \mathrm{~cm}$ (Macro), $50 \mathrm{~cm}$ $\sim \infty$; focus -2 steps, manual (Macro/Normal); shutter speed - 1/20 1/10000 sec (off mode); lens aperture F2,8; focal length $-5,23 \mathrm{~mm}$.

\subsection{Calibration of non-metric camera}

Determining camera inner orientation parameters two models were constructed [6]: 1) the use of two dimensions calibration grid (Fig 1) and particular software PhotoModeler; 2) the use of 3D-test field and software for self-calibration (Fig 2) [7].

The calibrations were performed within a time period of nine month.

For the first model eight photographs were taken from different angles of the dense point grid (calibrator slide): upper left, middle left, middle left vertical, bottom left, top right, middle right, middle right vertical and bottom right positions. It was very important to note that the grid should fill as much of the photographs as possible. The four control points are on the test grid and for easy identification these points has been marked by three circles for each control point ( 1 - no filled circles; 2 - one filled; 3 - two filled; 4 - three filled) (Fig 1). The constraint in size is that the all control points must appear in the photographs and must not be cut by edge of the photograph.

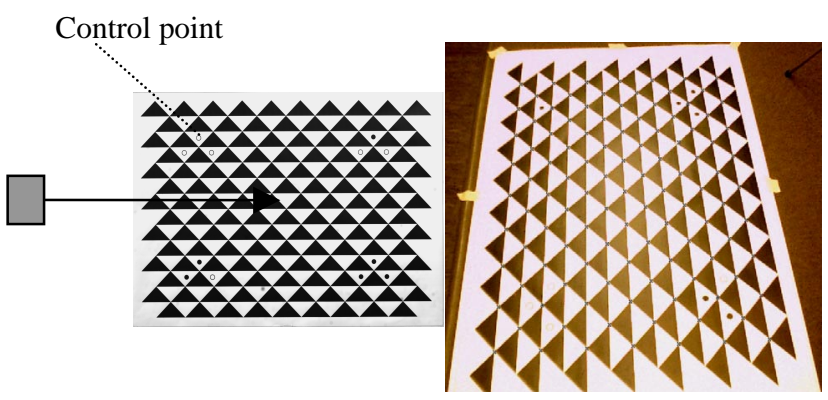

Fig 1. Calibration grid with control points and taking middle right vertical photo sets

For determining photo scale the distance between control points (1-4 or 2-3) on the calibrator grid pattern was measured as accurately as possible at the time the calibration pictures were taken. The experimental photography has been taken several times for getting better contrast and focus of photographs. A flash was not used to avoid wash the grid out and for excessive bright hot spots

The determination of digital camera format size was performed using appropriate method. It is sometimes difficult to find the correct format size of a digital camera's imaging chip. Only some cameras list the imagery size and even then the normal sizes vary between cameras and manufacturers.

The following technology can be used to obtain an approximate size for the imaging chip: make sure the camera is level and square to the wall; pattern of paper tape to a wall at a height of the camera; centre the pattern of paper in the viewfinder, move the camera in such a way that about $3 / 4$ of the image is taken; measure the distance $D$ from the pattern of paper to the location of the imaging chip (usually just behind the back of lens), after taking picture; measure the number of pixels taken up by the pattern of paper in the image $N_{x}, N_{y}$, using an image processing programme; determine the number of pixels in the whole image $S_{x}, S_{y}$.

The format size $W \times H$ (in the $x$ and $y$ directions) is calculated [7]:

$$
W=\frac{P_{x}}{D} \frac{c}{N_{x}} S_{x}, H=\frac{P_{y}}{D} \frac{c}{N_{y}} S_{y},
$$

where $P_{x}, P_{y}$ - the size of photographed pattern of paper in the $x$ and $y$ directions.

For the second model the 3D-test field with grid of targets was observed (Fig 2). Images of calibrator were taken in the laboratory and outdoors as well as using different means of light for getting better quality of photographs. All camera parameters, such as zoom, focus, aperture, etc were constant during data capture. Photo images have been received not of best quality (brightness, contrast) (Fig 2).

Parameters of camera calibration were calculated using software based on images relative orientation and block network adjustment with additional parameter. The stereo calibration was performed from 15 image pairs. In all cases 15 images were taken in different orientation of test field in order to capture all types of distortion.

The targets were used in automatic matching and for obtaining the highest measuring precision. The estimation process was based on 600 points observations of the 40 test field targets. The principal distance (focal length), coordinates of the principal point and the distortions has been determined.

Fig 3 shows the results of the interior orientation parameters determination for first and second models (M1, M2). The accuracy (RMSE) is about 0,25 pixels in the image plane.

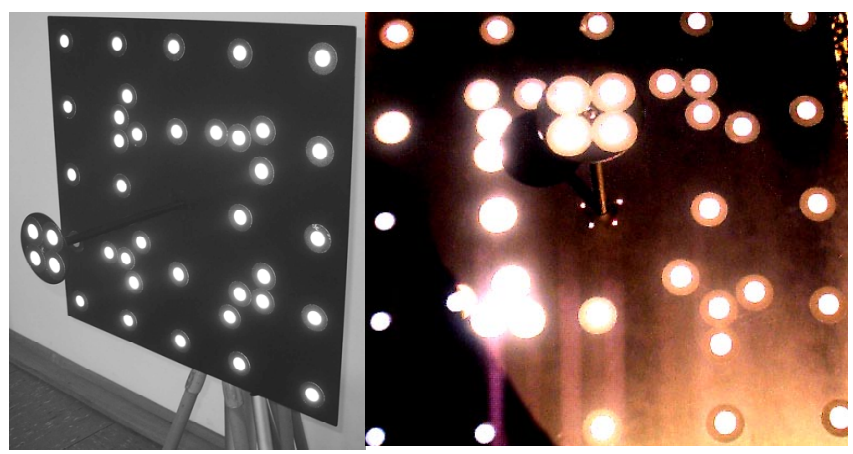

Fig 2. Images with calibration test field: used test field for calibration; image taken by amateur camera (one of calibrator sets) 

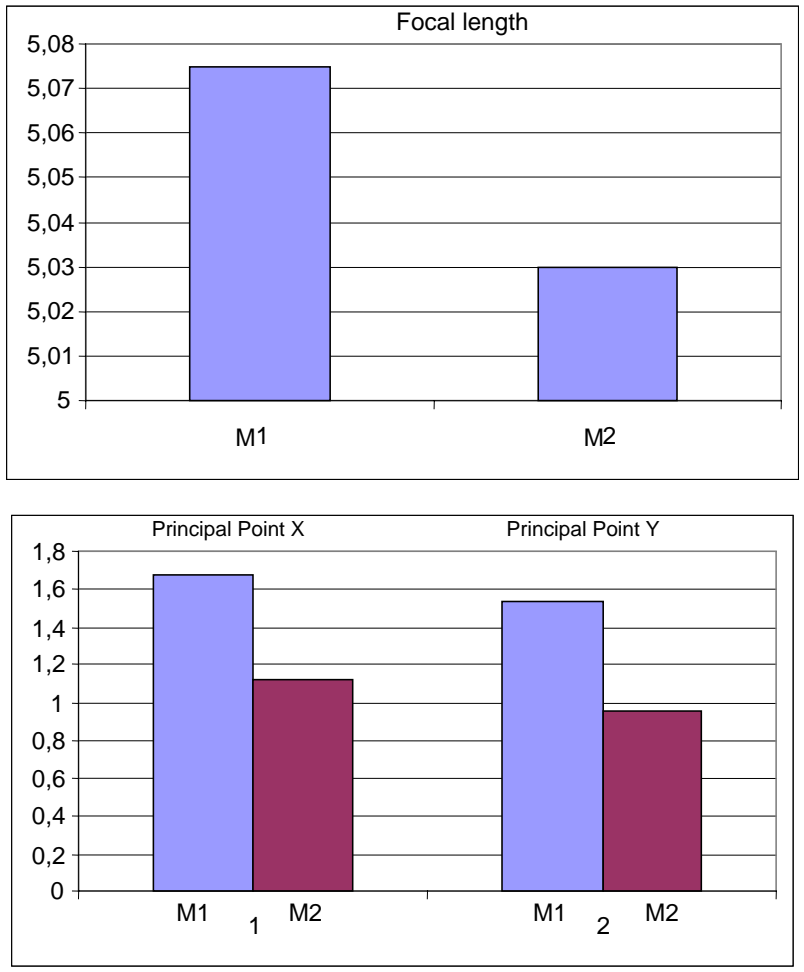

Fig 3. Results of cameras calibration: determined focal length and coordinates of principal point $(\mathrm{mm})$

The camera format size $W \times H(3,5139 \times 2,6791 \mathrm{~mm})$ has been calculated in model 1 , therefore pixel size is 3 $\mu \mathrm{m}$. The relative orientation of taken photographs was made by semi-automatic approach, because of not very enough focus of some grid points. Point's positions were corrected regarding to radial lens distortion $(K 1=$ $0,00282$ and $K 2=0,00042)$. Decentring distortion $(P 1$, $P 2)$ has been received a very small number, because corrections were not entered.

After comparison of received results it was defined that inner orientation parameters of investigated amateur camera varies about 30-50 pixels (about $0,1-0,15 \mathrm{~mm}$ ). Position of principal point is not stable (Fig 3).

\subsection{Performance evaluation by practical experiment}

In order to evaluate practical accuracy, experiment was performed taking stereo images by amateur digital camera of test field outdoors. 15 targets were set on the test field (Fig 4).

$3 \mathrm{D}$ coordinates of each target point were determined with accuracy: distance measurement $\pm 1 \mathrm{~mm}$; angle $\pm 2 "$. Stereo images were taken at a distance from the camera to the test field $Z=19,150 \mathrm{~m}$ and base line $B=6,579 \mathrm{~m}$ (base-height ratio 0,34 ).

The required (theoretical) mean-square errors of the object coordinates are calculated using formulae [8]:

$$
\sigma_{x y}=\frac{H}{c} \sigma_{p}, \sigma_{z}=\frac{H}{c} \frac{H}{B} \sigma_{p},
$$

where $\sigma_{p}$ - pointing accuracy.

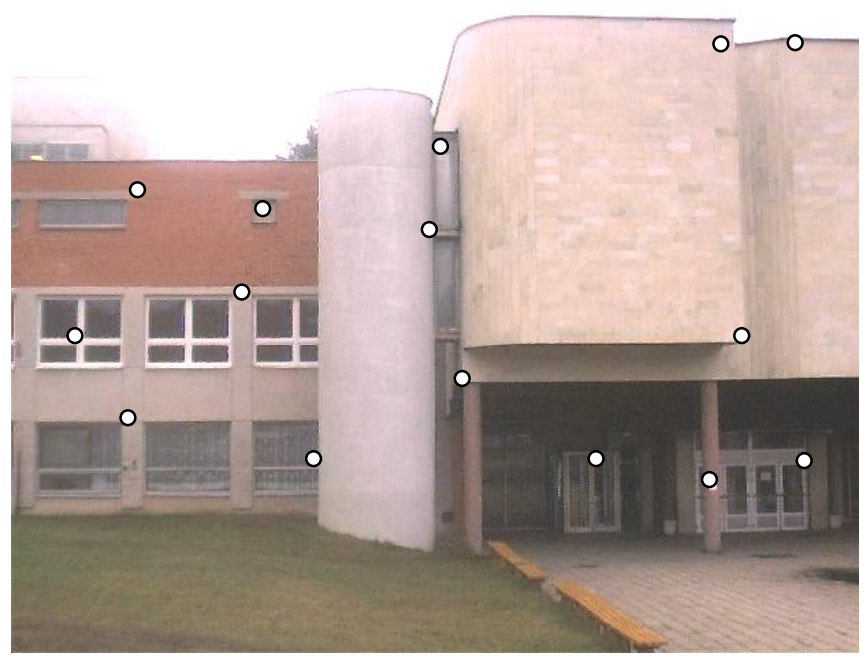

Fig 4. Outdoor test field

The results of practical experiment on performance evaluation of digital amateur camera are:

- Determined root mean square error (RMSE) for target (check) points:

$$
\sigma_{\mathrm{xy}}=8,1 \mathrm{~mm} ; \sigma_{\mathrm{z}}=15,9 \mathrm{~mm} \text {. }
$$

- Theoretical RMSE computed using equation (7):

$$
\sigma_{\mathrm{xy}}=3,3 \mathrm{~mm} ; \sigma_{\mathrm{z}}=9,6 \mathrm{~mm} \text {, as } 0,3 \text { pixel }
$$
pointing accuracy was assumed.

This result is improved by radial distortion using determined inner orientation parameters by calibration. The accuracy for the amateur, low-resolution digital camera comparing with theoretical accuracy has been received not sufficient photogrammetric application. However, it needs further investigations correcting results by radial and tangential distortions. Such amateur camera can be used in photogrammetric field only for, eg buildings documentation, etc, because it cannot promise an accuracy of convenient photogrammetry measurements.

\section{Conclusions}

It is expected that amateur digital cameras can be widely used in photogrammetric field as an essential tool, which is able to advance traditional photogrammetric application.

Investigation of camera inner orientation parameters instability shows that it varies about 30-50 pixels (about $0,1-0,15 \mathrm{~mm})$. Position of principal point is not stable. Because non-metric cameras are of short focal length it needs for accurate calibration using larger test field. The cameras instability requires the calibration before each project. Corresponding to relationship of camera parameters determination with pixel numbers, it can be defined that the investigated camera is not sufficient for getting better quality images.

From the performance evaluation it can be concluded that amateur digital camera under certain, limited accuracy requirements can be used for photogrammetric purposes. The investigations need to be extended by taking images with lager resolution (more 
than 3 mega pixels), fixing focus cameras in mobile phones, creating more flexible distortion models, etc

The investigated low-cost, low-resolution digital camera does not meet the accuracy requirements of digital photogrammetry, therefore it can be widely used for low-accuracy photogrammetric application.

\section{References}

1. Butowtt, Jerzy; Kaczynski, Romuald. Fotogrametria. Warszawa: Wojskowa Akademia Techniczna, 2003. 375 p. (in Polish).

2. Kraus, Karl. Photogrammetry. Advanced methods and applications. Vol 2. Ummler/Bonn, 1997. 466 p.

3. Santiago, Cruz; Jares, Cardenal; Jorge, Delgado. A program for automatic inner orientation of digitized nonmetric images. International Archives of Photogrammetry and Remote Sensing, Vol XXXIII, Part 5/1. Amsterdam, The Netherlands, 2000, p. 149-156.

4. Charles, Toth. Experiences with frame CCD arrays and direct georeferencing. In: Proceedings of Photogrammetric Week, Stuttgart, 1999, p. 95-107.

5. Abraham, Steffen. Test field based self-calibration of a Stereo Camera System. In: Proceedings of Institute of Bonn University, 1998, p. 3-13.

6. Ruzgienè, B. Determination of inner orientation parameters of digital camera. Geodesy and Cartography
(Geodezija ir kartografija), Vol XXVIII, No 2, Vilnius: Technika, 2002, p. 45-49 (in Lithuanian).

7. Labe, T.; Förstner, W. Geometric stability of low-cost digital consumer camera. International Archives of Photogrammetry and Remote Sensing, Vol XXXV, Part B, Istanbul, Turkey, 2004, p. 528-535.

8. Manual of Photogrammetry (ed. by Chris McGlone). Fifth ed. American Society for Photogrammetry and Remote Sensing, USA, 2004. 1151 p.

Birutė RUZGIENĖ. Associate Professor, Doctor.

Vilnius Gediminas Technical University, Dept of Geodesy and Cadastre, Saulètekio al. 11, LT-10223 Vilnius-40, Lithuania (tel +370 5 2744703, fax +370 5 2744705), e-mail: birute.ruzgiene@ap.vtu.lt.

A graduate of Vilnius Civil Engineering Institute (engineer of geodesy, 1968). Doctor (Vilnius Gediminas Technical University, 1999). Research training at Moscow Institute of Geodesy, Aerosurveying and Cartography (1986), at Norway AO Fjellanger Widerøe (1995), at Warsaw Institute of Geodesy and Cartography (1998), at Photogrammetry Institute of Bonn University (2000). Author of more than 30 scientific papers.

Research interests: digital cartography, aerophotogrammetry. 